\title{
Analysis of rRNA synthesis using quantitative transcription run-on (qTRO) in yeast
}

\author{
Michal Koper ${ }^{\star, 1}$ \& Seweryn Mroczek ${ }^{1}$ \\ ${ }^{1}$ Institute of Genetics \& Biotechnology, Faculty of Biology, University of Warsaw, A. Pawinskiego 5a, 02-106, \\ Warsaw, Poland
}

BioTechniques 65: 163-168 (September 2018) 10.2144/btn-2018-0073

Keywords: quantitative transcription run-on (qTRO) • ribosomal RNA • RNA polymerase I

Comparative transcriptional analyses require appropriate and precise normalization. Here we describe a modified transcription run-on (TRO) method, named quantitative TRO (qTRO), that allows quantification of nascent transcription activity. The most critical improvement it introduces is a new standardization method for RNA isolation and hybridization steps, enabling transcription activity quantification and comparative biological analysis. We used this technique with chromatin immunoprecipitation to investigate RNA polymerase I (RNAPI) transcription activity and its rDNA gene profiles in Saccharomyces cerevisiae. We designed a set of new oligonucleotide probes complementary to nascent ribosomal RNA ( $\mathrm{RRNA}$ ) transcripts and standardized their hybridization strength. The qTRO method could be successfully implemented to study RNAPI transcription in response to oxidative stress and in two mutant strains with impaired rRNA synthesis.

One of the most widely used techniques to investigate nascent transcription activity is RNA polymerase chromatin immunoprecipitation (ChIP) or ChIP sequencing, which allows the mapping of polymerase densities along the targeted genes but may not directly reflect transcription activity in regions where the enzyme pauses. Subsequently, the transcription run-on (TRO) method allows the measurement of nascent transcript synthesis.

The classical TRO technique relies on nascent transcript labeling during transcriptional pulse, followed by total RNA isolation and labeled target detection. TRO approaches in yeast are based on sarkosyl detergent treatment that permeabilizes cell and nuclear membranes to efficiently deliver ${ }^{32} \mathrm{P}$-labeled UTP into the nucleus [1-4]. After transcriptional pulse, total RNA is isolated, partially fragmented, hybridized to gene-specific probes (M13 ssDNA, oligonucleotides, denatured plasmids, or in vitro transcribed RNA) immobilized on a nylon membrane.
After hybridization, quantitative signal analysis demonstrates the transcription activity of individual genes and identifies transcription termination defects, such as read through [5-7].

Total transcription rates are measured by techniques that utilize radioactive labeling of nucleic acids in vivo during culture, but they do not allow the analysis of polymerase activity on specific transcription units [8]. Conversely, recent developments in highthroughput sequencing methods and nucleotide chemistry, including global run-on, native elongating transcript sequencing, and precision nuclear run-on sequencing, have enabled several genome-wide applications of TRO in yeast and the quantification of RNA polymerase transcription rates [9-12]. Although these approaches have revolutionized research on transcription, their use is still limited because of their labor intensiveness and high cost.

Despite the many advantages of the TRO method, additional controls are required to increase its analytical and comparative potential. This method typically does not directly compare the transcription levels obtained in different experimental conditions or strains because of technical limitations. Therefore, we developed a modified quantitative TRO (qTRO) technique to ensure repeatability and reliability of obtained measurements of nascent transcription activity and validated it on RNA polymerase I (RNAPI) transcription.

rRNA-encoding genes in yeast are clustered in an 1-2 Mb region comprising $\leq 200$ tandem copies of the rDNA locus (9.1 kb each) located on the right arm of chromosome XII. The region is transcribed by RNAPI as 35S pre-rRNA precursor molecules, which are subsequently exoand endo-nucleolytically processed into mature 18S, 5.8S and 25S rRNA (Figure 1A; reviewed in $[13,14])$. rRNA synthesis constitutes approximately $60 \%$ of all transcription activity in the nucleus, making it an excellent model to validate our modified qTRO approach [15].

\section{METHOD SUMMARY}

Our quantitative transcription run-on (qTRO) protocol involved: i) the collection of equal number of cells for each condition; ii) addition of the exogenous, in vitro transcribed and radiolabeled spike-in RNA to each sample during cell homogenization; iii) utilization of the oligonucleotide probe complementary to the spike-in RNA for monitoring RNA recovery by comparing hybridization signals between blots; and iv) correction of the hybridization signal strength for 75-80-nt probes complementary to different regions of the rRNA by hybridization to in vitro transcribed and radiolabeled 35S rRNA precursors. 
(A)

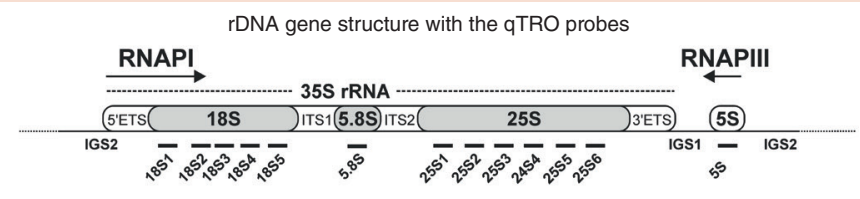

(B)

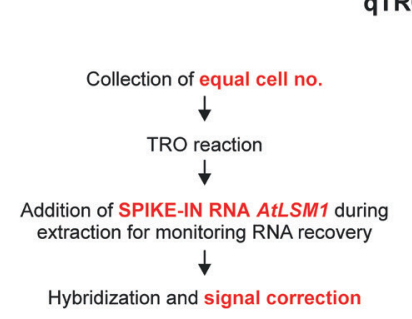

qTRO PRINCIPLES

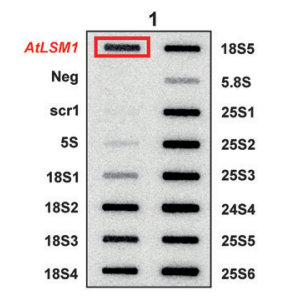

Standardization of the probe signals and RNAPI transcription profile

(C)

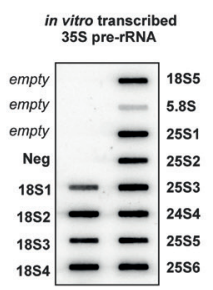

(D)

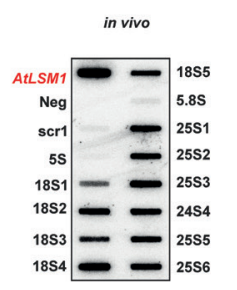

A summary of the qTRO protocol and representative blots are shown in Figure 1B. Collecting equal number of cells is crucial for this technique. We strongly

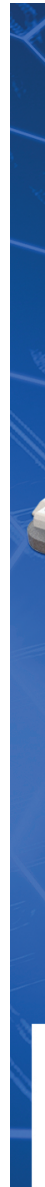

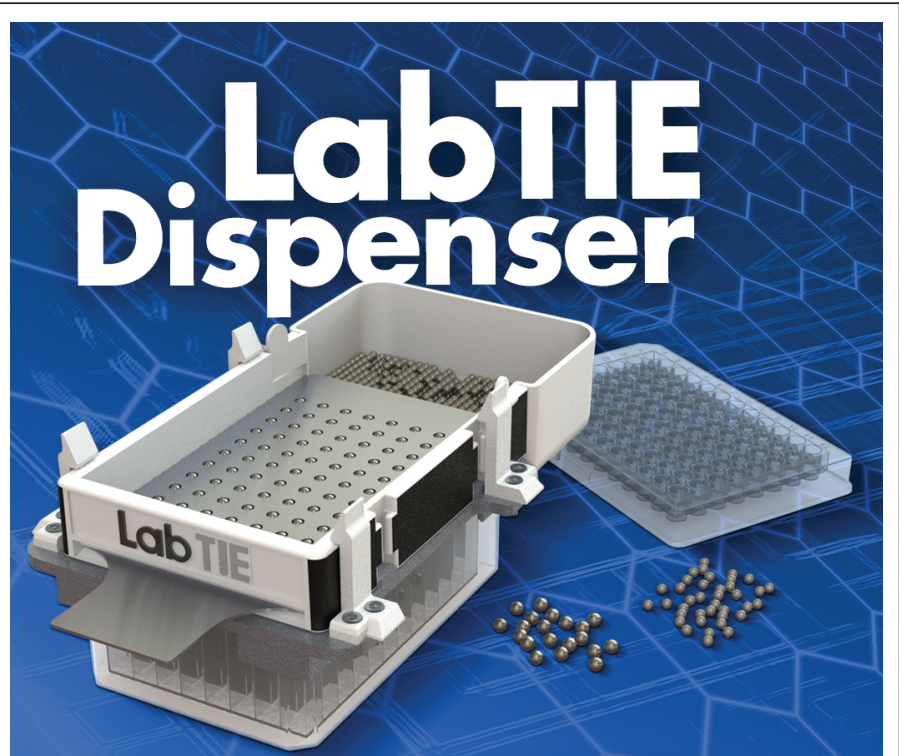

- EASILY DISPENSE BEADS, POWDERS INTO MICROPLATES AND TUBES

- ECONOMICAL VS PRICEY PRE-FILLED LYSIS TUBES

- ACCURATE, FAST, SIMPLE, CUSTOMIZABLE

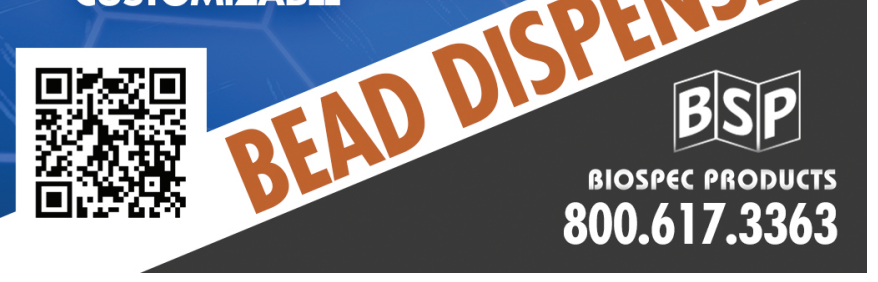

recommend checking the correlation of the actual cell number with optical density of cultures measured at $600 \mathrm{~nm}$ wavelength $\left(\mathrm{OD}_{600}\right)$ using a Thoma cell counting chamber. The optimal $\mathrm{OD}_{600}$ range for $50-\mathrm{ml}$ cultures is $0.2-0.4$. To simplify the procedure for multiple analyses, we decided to use standard curves of cell numbers at the same $\mathrm{OD}_{600}$ range that were generated before the qTRO experiments and collect different volumes of culture to maintain consistent cell numbers. In cases where a single strain needs to be analyzed after a brief change in growth conditions, collection of cultures with equal $\mathrm{OD}$ is acceptable. In qTRO, we decided to not include in-cell control to monitor cell lysis efficiency, which may depend on many factors, including the cell shape and cell wall thickness. However, the protocol can be expanded to include in-cell controls for monitoring homogenization,
Figure 1. Principles of qTRO and RNAPI activity profile. (A) The rDNA gene structure diagram with marked positions of probes designed for qTRO. The individual rDNA unit is composed of the RNAPI transcribed gene encoding 35S rRNA, which is the precursor to the mature 18S, 5.8S and 25S rRNA, separated by internal transcribed spacers (ITS1 and ITS2). 35S rRNA is flanked by $5^{\prime}$ and $3^{\prime}$ external transcribed spacers (ETSes) and the intergenic spacers IGS1 and IGS2. Each unit is separated by RNAPIII-transcribed 5S rRNA gene. Arrows indicate the direction of RNAPI and RNAPIII transcription. (B) The outline of qTRO approach principles and two exemplary hybridization results. Signals for AtLSM1 probe used as RNA spike-in controls are marked with red frames. (C, D) Oligonucleotide probes standardization and wild-type RNAPI transcription profile. (C) Representative blot used for correcting the hybridization strength of oligonucleotide probes (hybridization with in vitro transcribed, [ $\left.\alpha^{32} \mathrm{P}\right]$ UTP-labeled $35 \mathrm{~S}$ pre-rRNA) and their in vivo signals in Rpa34-13myc-tagged W303 strain. (D) Comparison of the in vitro probe signals with uncorrected in vivo and corrected RNAPI transcription profile. Data are presented as signals normalized to the 18S1 probe and represent mean values of three biological replicates with standard deviations. Yeasts were grown in liquid YPD medium (yeast extract, peptone, $2 \%$ glucose) at $30^{\circ} \mathrm{C}, 45 \mathrm{ml}$ of 0.35 $\mathrm{OD}_{600}$ cultures were collected.

qTRO: Quantitative transcription run-on; RNAPI: RNA polymerase I; RNAPIII: RNA polymerase III.

including genomic DNA detection using QPCR of interphase material obtained during the phenol/GTC extraction of RNA [16]. However, omitting this control results in a simpler protocol that is much easier to perform due to the high radioactivity of the preparations obtained.

To standardize RNA isolation and subsequent hybridizations, synthetic ${ }^{32} \mathrm{P}$ labeled spike-in control transcript of the LSM1 fragment from Arabidopsis thaliana was used, namely AtLSM1 [16,17]. Equal amounts of in vitro transcribed AtLSM1 (500 counts per second [cps]) were added to each single qTRO reaction before RNA extraction from cells. Thus, comparing the hybridization signal for the AtLSM1 probe between blots in one experimental set allowed us to measure the correction factors of RNA isolation efficiency for individual blots and calculate TRO hybridization values for all probes used (Figure 1B).

Next, we designed a set of 16 oligonucleotide probes (Figure 1A, Supplementary Tables S1 and S2). To limit the possibility of secondary structure formation, we used sequences with a GC content of $<60 \%$ and relatively high $A$ content as they may preferentially pair with ${ }^{32} \mathrm{P}$-labeled $\mathrm{U}$ in the nascent transcript. 12 of the 16 probes were complementary to 
mature rRNA species derived from the primary RNAPI transcript (five for 18S, one for $5.8 \mathrm{~S}$ and six for $25 \mathrm{~S}$ rRNA). The probe set also included additional oligonucleotides complementary to the SCR1 and 5S rRNA, RNAPII and RNAPIII transcripts respectively, and the standardization AtLSM1 probe. Moreover, the agaA transcript from Aspergillus nidulans served as a negative control [18]. BLAST searches of the $S$. cerevisiae genome revealed no target sequences for the aga $A$ probe; thus, it was used for background correction. The value of the aga $A$ hybridization signal was subtracted from the obtained signals of other probes in the set.

Importantly, we standardized the signals of 18S, $5.8 \mathrm{~S}$ and $25 \mathrm{~S}$ rRNA probes. When calibrating using classical $\mathrm{TRO}$, the obtained signals are divided by the number of ${ }^{32} \mathrm{P}$-labeled $U$ in the target sequence of the nascent transcript. However, this did not reveal the hybridization strength of individual probes. In our qTRO protocol, probe signals were standardized against in vitro transcribed $\left[\alpha^{32} \mathrm{P}\right]$ UTP-labeled 35S rRNA. First, we optimized the in vitro transcription reaction conditions to increase T7 RNA polymerase processivity as we observed several preterminated transcripts under the standard assay conditions applied to AtLSM1. Performing the reaction at $16^{\circ} \mathrm{C}$ and altering the proportion of the limiting nucleotide (UTP) to other nucleotides, resulted in a significant increase in T7 RNA polymerase processivity (Supplementary Figure S1A). Although not all polymerases reach the run-off position in the template, two major $~ 6.5-\mathrm{kb}$ RNAs are detected using both the most proximal (18S1) and distal (25S6) probes for 35S rRNA, as verified by northern blotting (Supplementary Figure S1B). Next, in vitro transcribed $35 \mathrm{~S}$ rRNA (1500 cps) was processed similarly to RNA isolated from cells after ${ }^{32} \mathrm{P}$-labelling. To reflect the conditions during the RNA fragmentation and hybridization in qTRO, hot $35 \mathrm{~S}$ rRNA was mixed with approximately $500 \mu \mathrm{g}$ of carrier-unlabeled total RNA, corresponding to the amount isolated from 10-20 OD ${ }_{600}$ units of cells using the phenol/GTC method. Then it was treated with $\mathrm{NaOH}$ and hybridized with probes. Hybridization strength factors for each 35S rRNA probe (Supplementary Table S2) were calculated using the obtained signals (Figure 1C); the values were used to correct the final in vivo TRO signals (Figure 1D). The obtained data were used to model 35S RNA transcription profiles, which revealed two distinct transcription peaks of similar amplitude in $18 S$ and $25 S$ rRNA (Figure 1D). A substantial decrease in RNAPI activity within the 5.8S rRNA-coding region could be explained by co-transcriptional cleavage of the pre-rRNA at the A2 site in ITS1 [19]. Another possible explanation is an increase in R-loop (RNA/DNA hybrids) formation, demonstrated by ChIP-qPCR and reflected in the decreased RNAPI occupancy over ITS1-ITS2, as observed by electron microscopy [20]. Subsequently, we investigated RNAPI occupancy over rDNA using a c-myc-tagged Rpa34 subunit and ChIP-qPCR, similar to that in previous studies [7,20]. The density distribution profile of RNAPI over the rDNA correlated with the transcription profile obtained by the qTRO approach (Figures 1D and 2A).

We investigated the possibility of conducting an efficient qTRO using flashfrozen cells, which would introduce a safe stopping point for the experiment, similar to that in biotin-genomic run-on [21]. We found that hybridization signals reduced $>30 \%$ for qTRO performed using flashfrozen cells compared with qTRO performed using fresh harvested cells (Supplementary Figure S2). Therefore, we recommend avoiding freezing when working with strains or growth conditions that result in low RNAPI transcription levels and proceeding immediately with qTRO.

Next, to assess the suitability of the qTRO method in comparative biological experiments, we applied it to analyze 35S rRNA transcription under oxidative stress conditions, wherein rDNA and ribosomal protein-coding genes transcription inhibition is expected [22]. To compare the results obtained using qTRO and ChIP-qPCR methods, experiments were performed using a c-myc-tagged Rpa34 RNAPI subunit in a W303 background. As expected, the qTRO method demonstrated a significant $(\leq 60 \%)$ decrease in RNAPI transcription activity within 5 min of oxidative stress (Figure 2B). Similarly, the observed qTRO rRNA transcription profile reflected RNAPI occupancy over the rDNA, as mapped by ChIP-qPCR (Figure 2A). Thus, the decrease in transcription under oxidative stress conditions results from polymerase complex dissociation
The Geno/Grinder ${ }^{\circledR}$ is a high-throughput plant and animal tissue homogenizer specifically designed for rapid cell disruption, lysis and tissue homogenization.

\section{- Adjustable clamp that} accommodates up to six deep-well titer plates, or multiple sample tubes from $2 \mathrm{~mL}$ to $50 \mathrm{~mL}$.

\section{- Programmable touch screen control panel that saves up to 500 protocols.}

(+1) 732-623-0465

learnmore@spex.com

SPEXSAMPLEPREP.COM/GENOGRINDER 
(A)

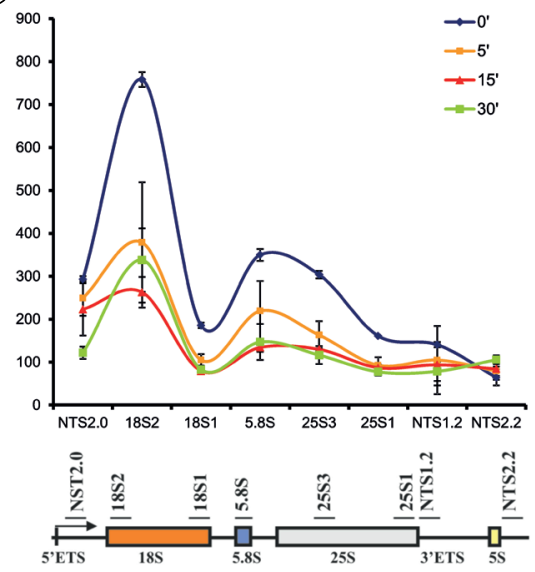

(C)

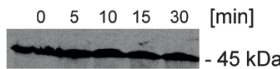

(B)
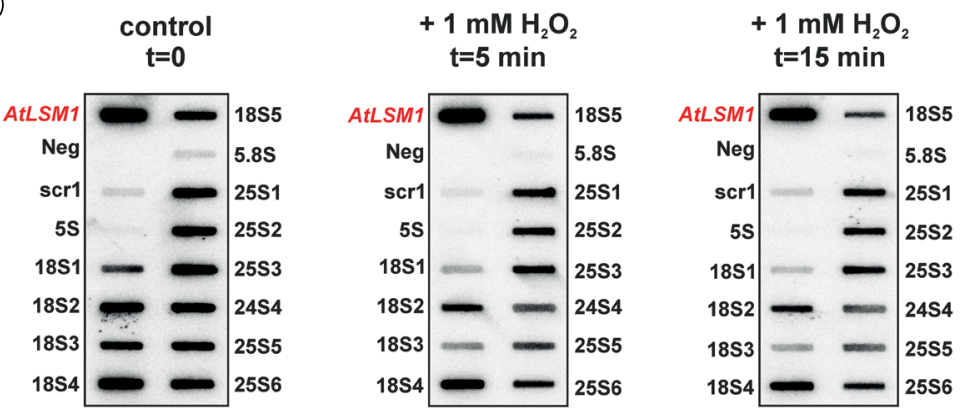

RNAPI transcription profiles
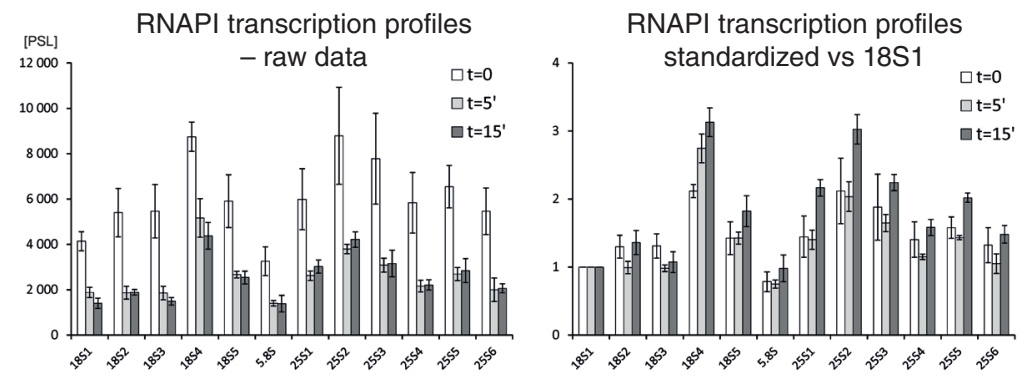

Figure 2. Decrease in rRNA transcription correlates with RNAPI dissociation from the template upon oxidative stress. (A) ChIP assay revealing loss of RNAP association on rDNA in yeast cells after induction of oxidative stress. Relative RNAPI occupancy over rDNA gene in cells treated with $1 \mathrm{mM} \mathrm{H}_{2} \mathrm{O}_{2}$ for 5,15 and $30 \mathrm{~min}$. Graph shows mean values of three biological replicates with standard deviations, which were corrected for background. qPCR primer positions are denoted on the rDNA scheme. The Rpa34-13myc-tagged strain was grown in YPD medium at $30^{\circ} \mathrm{C}$ and treated with $1 \mathrm{mM} \mathrm{H} \mathrm{H}_{2}$ up to 30 min. (B) Oxidative stress response involves strong RNAPI inhibition, as revealed by qTRO. Representative qTRO data obtained for control and cells treated with 1 mM $\mathrm{H}_{2} \mathrm{O}_{2}$ for 5 and 15 min. Absolute hybridization signals (corrected vs AtLSM1 control signal and probe hybridization strength) in photo-stimulated luminescence units and standardized data presented as signal normalized to the 18S1 probe are shown. Bars represent mean values of three biological replicates with standard deviations. Strain and growth conditions as on panel A, $45 \mathrm{ml}$ of $0.35-\mathrm{OD}_{600}$ cultures were collected. (C) Western blot analysis of Rpa34-13myc protein level with anti-myc tag antibodies after inducing oxidative stress.

ChIP: Chromatin immunoprecipitation; qTRO: Quantitative transcription run-on; RNAPI: RNA polymerase I.

from the template rather than a decrease in the steady state levels of Rpa34, as verified by western blotting (Figure 2C).

Finally, to fully validate our method, we used our qTRO approach to evaluate the RNAPI transcription activity in a rpa12 $\Delta$ strain with elevated RNAPI processivity and in spt4 $\Delta$ with decreased rRNA transcription. Rpa12, a subunit of RNAPI, has intrinsic RNA cleavage activity and shares homology to the small subunit Rpb9 of RNAPII and Rpc11 of RNAPIII, all of which are involved in transcription termination. Deletion of the RPA12 gene leads to minor growth effects but no pre-rRNA processing defects; however, a visible increase in RNAPI processivity is manifested by elevated transcription levels and altered RNAPI termination [5]. While measuring the RNAPI transcription activity in qTRO, we observed an average of an approximately 6.1-fold increase in $35 S$ rRNA synthesis in the rpa12 $\Delta$ strain compared with that in the WT strain, with a maximal signal change of approximately 12-fold for the 5.8S rRNA probe (Supple- mentary Figure S3A). Except for changes in the 5.8S rRNA-coding region, the overall RNAPI transcription profile in the mutant resembles that in the WT. Our data are consistent with previous observations suggesting a role of Rpa12 in polymerase fidelity control and demonstrating that its absence results in increased RNAPI velocity [5].

The DRB sensitivity-inducing factor (DSIF) complex is composed of Spt4 and Spt5 and serves as a transcription elongation factor for both RNAPI and RNAPII. DSIF binds ssRNA, has diverse functions in transcription regulation (elongation, silencing, RNA processing and quality control), and is engaged in transcription-coupled DNA repair. Deletion of the SPT4 gene results in minor growth effects, temperature sensitivity at $37^{\circ} \mathrm{C}$, and pre-rRNA processing defects. A previous study has demonstrated that the total RNA synthesis rate in spt4 $\Delta$ is approximately $70 \%$ of that in the WT strain [23]. Consistent with these findings, our qTRO data showed that in
spt4A mutants, RNAPI transcription is reduced to approximately $61 \%$ of that in the WT strain (Supplementary Figure S3B), but the overall RNAPI transcription profile remains unchanged.

Here we reported a new method for calibrating and quantifying yeast TRO that we successfully validated by using it to compare RNAPI transcription levels under different biological conditions and in different mutants. This modification of the TRO protocol allows rapid and direct comparison of transcription levels between mutants or conditions of interest. The results obtained by qTRO will serve as adequate preliminary results for further verification by complementary methods. The detailed qTRO protocol is available with this article.

\section{Supplementary data}

To view the supplementary data that accompany this paper please visit the journal website at: www.future-science. com/doi/suppl/10.2144/BTN-2018-0073 


\section{Author contributions}

MK conceived the project. MK designed and performed the majority of experiments including all qTRO analyses. SM performed ChIP-qPCR experiments and optimized 35SrRNA transcription in vitro. MK and SM wrote the article.

\section{Acknowledgments}

We would like to thank Joanna Kufel at the Institute of Genetics and Biotechnology, Faculty of Biology, University of Warsaw, for advice and inspiration; Bernhard Dichtl and Agnieszka Wengi at the Institute of Molecular Life Sciences, University of Zurich, for training MK in the TRO technique (BD present address: Centre for Cellular and Molecular Biology, School of Life and Environmental Sciences, Deakin University); and Aziz El Hage at the Wellcome Trust Centre for Cell Biology, University of Edinburgh, for training SM in ChIP-qPCR technique and for the Rpa34-13myc yeast strain. We thank David Tollervey at the Wellcome Trust Centre for Cell Biology, University of Edinburgh for providing the plasmid bearing 355 rDNA.

\section{Financial \& competing interests disclosure}

This work was supported by the National Science Centre, Poland grant [N N301 314537] (to MK), Ministry of Science and Higher Education, Poland 'luventus' Plus grant [IP2012 046272] and FEBS Collaborative Experimental Scholarships for Central and Eastern Europe (to SM). Experiments were performed with CePT infrastructure financed by the European Regional Development Fund allocated by the European Union [Innovative economy 2007-13, Agreement POIG.02.02.00-14-024/0800]. The authors have no other relevant affiliations or financial involvement with any organization or entity with a financial interest in or financial conflict with the subject matter or materials discussed in the manuscript apart from those disclosed. Writing support was provided by Enago (Crismon Interactive Inc.) scientific editing service to improve quality of the manuscript.

\section{Open access}

This work is licensed under the Creative Commons Attribution 4.0 License. To view a copy of this license, visit http://creativecommons.org/licenses/by/4.0/

\section{References}

1. Elion EA, Warner JR. An RNA polymerase I enhancer in Saccharomyces cerevisiae. Mol. Cell. Biol. 6(6), 2089-2097 (1986).

2. Maundrell K. nmt1 of fission yeast. A highly transcribed gene completely repressed by thiamine. J. Biol. Chem. 265(19), 10857-10864 (1990).

3. Warner JR. Labeling of RNA and phosphoproteins in Saccharomyces cerevisiae. Methods Enzymol. 194, 423-428 (1991).

4. Birse CE, Lee BA, Hansen K, Proudfoot NJ. Transcriptional termination signals for RNA polymerase II in fission yeast. EMBO J. 16(12), 3633-3643 (1997).

5. Prescott EM, Osheim YN, Jones HS et al. Transcriptional termination by RNA polymerase I requires the small subunit Rpa12p. Proc. Natl Acad. Sci. USA 101(16), 6068-6073 (2004).

6. Kawauchi J, Mischo H, Braglia P, Rondon A, Proudfoot NJ. Budding yeast RNA polymerases I and II employ parallel mechanisms of transcriptional termination. Genes Dev. 22(8), 1082-1092 (2008).

7. El Hage A, Koper M, Kufel J, Tollervey D. Efficient termination of transcription by RNA polymerase I requires the $5^{\prime}$ exonuclease Rat1 in yeast. Genes Dev. 22(8), 1069-1081 (2008)

8. Fraser RS, Carter BL. Synthesis of polyadenylated messenger RNA during the cell cycle of Saccharomyces cerevisiae. J. Mol. Biol. 104(1), 223-242 (1976)

9. García-Martínez J, Aranda A, Pérez-Ortín JE. Genomic run-on evaluates transcription rates for all yeast genes and identifies gene regulatory mechanisms. Mol. Cell. 15(2), 303-313 (2004).

10. Churchman LS, Weissman JS. Native elongating transcript sequencing (NET-seq). Curr. Protoc. Mol. Biol. Chapter 4, Unit 4.14.1-17 (2012).

11. Booth GT, Wang IX, Cheung VG, Lis JT. Divergence of a conserved elongation factor and transcription regulation in budding and fission yeast. Genome Res. 26(6), 799-811 (2016).

12. Mena A, Medina DA, García-Martínez J et al. Asymmetric cell division requires specific mechanisms for adjusting global transcription. Nucleic Acids Res. 45(21), 12401-12412 (2017).

13. Turowski TW, Tollervey D. Cotranscriptional events in eukaryotic ribosome synthesis. Wiley Interdiscip. Rev. RNA. 6(1), 129-139 (2015).

14. Fernández-Pevida A, Kressler D, de la Cruz J. Processing of preribosomal RNA in Saccharomyces cerevisiae. Wiley Interdiscip. Rev. RNA. 6(2), 191-209 (2015).

15. Warner JR. The economics of ribosome biosynthesis in yeast. Trends Biochem. Sci. 24(11), 437440 (1999).

16. Alexander RD, Barrass JD, Dichtl B et al. RiboSys, a high-resolution, quantitative approach to measure the in vivo kinetics of pre-mRNA splicing and $3^{\prime}$-end processing in Saccharomyces cerevisiae. RNA N. Y. N. 16(12), 2570-2580 (2010).

17. Alexander RD, Innocente SA, Barrass JD, Beggs JD. Splicing-dependent RNA polymerase pausing in yeast. Mol. Cell. 40(4), 582-593 (2010).

\section{ali-Q $\mathbf{Q}^{\mathrm{m}}$}

\section{The world's only} aliquoting pipet controller

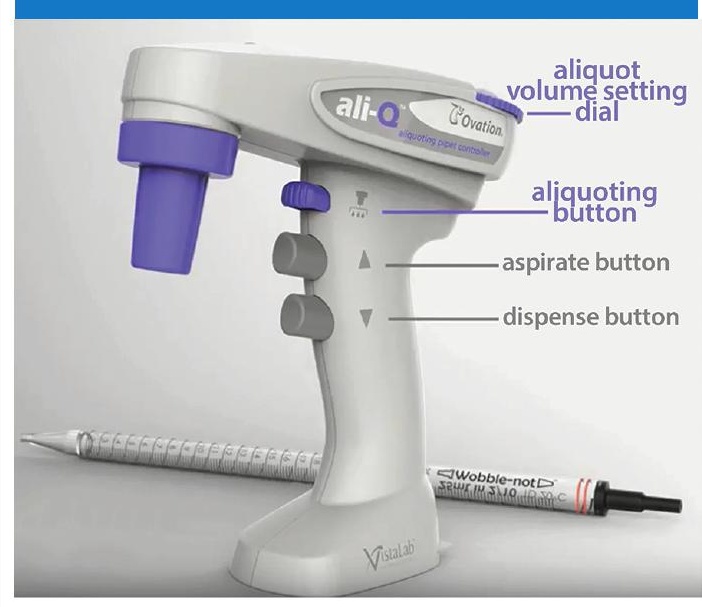

\section{Accurate Repeat Pipetting}

Multidispense the same volume over and over again with high accuracy \& precision, without having to

"eyeball" the meniscus

\section{Recent review*}

"Great for dispensing the same amount repeatedly..... You can concentrate on the tip of the pipette and the culture plates because you don't have to watch the graduations on the pipette."

\section{There is no other pipetter like ali- $Q$} ali-Q's innovative intelligent measuring system means:

- Use with serological pipets of any size or brand.

- Arm/pipet angle does not affect accuracy \& precision.

- Accurate repeat pipetting using a single button.

Also use ali- $Q$ to aspirate and dispense as with your traditional, non-aliquoting pipet controllers/fillers

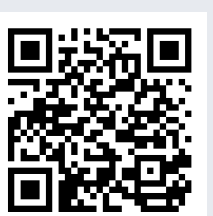

See the ali- $Q^{\mathrm{TM}}$ in action: vistalab.com/ali-c

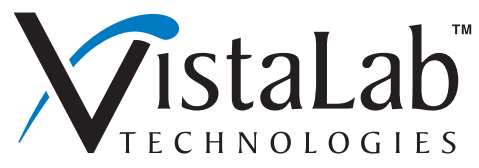

Think Differently. Feel Better.

vistalab.com/ali-q • +1 914-244-6226

2 Geneva Road, Brewster NY 10509 
18. Borsuk P, Dzikowska A, Empel J, Grzelak A, Grześkowiak R, Weglenski P. Structure of the arginase coding gene and its transcript in Aspergillus nidulans. Acta Biochim. Pol. 46(2), 391403 (1999).

19. Kos M, Tollervey D. Yeast pre-rRNA processing and modification occur cotranscriptionally. Mol. Cell. 37(6), 809-820 (2010).

20. El Hage A, French SL, Beyer AL, Tollervey D. Loss of Topoisomerase I leads to R-loopmediated transcriptional blocks during ribosomal RNA synthesis. Genes Dev. 24(14), 1546-1558 (2010).

21. Jordán-Pla A, Miguel A, Serna E, Pelechano V, Pérez-Ortín JE. Biotin-Genomic Run-On
(Bio-GRO): a high-resolution method for the analysis of nascent transcription in yeast. Methods Mol. Biol. Clifton NJ. 1361, 125-139 (2016).

22. Gasch AP, Spellman PT, Kao CM et al. Genomic expression programs in the response of yeast cells to environmental changes. Mol. Biol. Cell. 11(12), 4241-4257 (2000).

23. Schneider DA, French SL, Osheim YN et al. RNA polymerase II elongation factors Spt $4 p$ and Spt5p play roles in transcription elongation by RNA polymerase I and rRNA processing. Proc. Natl Acad. Sci. USA 103(34), 12707-12712 (2006).
First draft submitted: 22 May 2018;

Accepted for publication: 9 July 2018

Address correspondence to: Michal Koper; Institute of Genetics \& Biotechnology, Faculty of Biology, University of Warsaw, A. Pawinskiego 5a, 02-106, Warsaw, Poland; E-mail: mkoper@igib.uw.edu.pl

\section{To purchase reprints of this article contact:} s.cavana@future-science.com

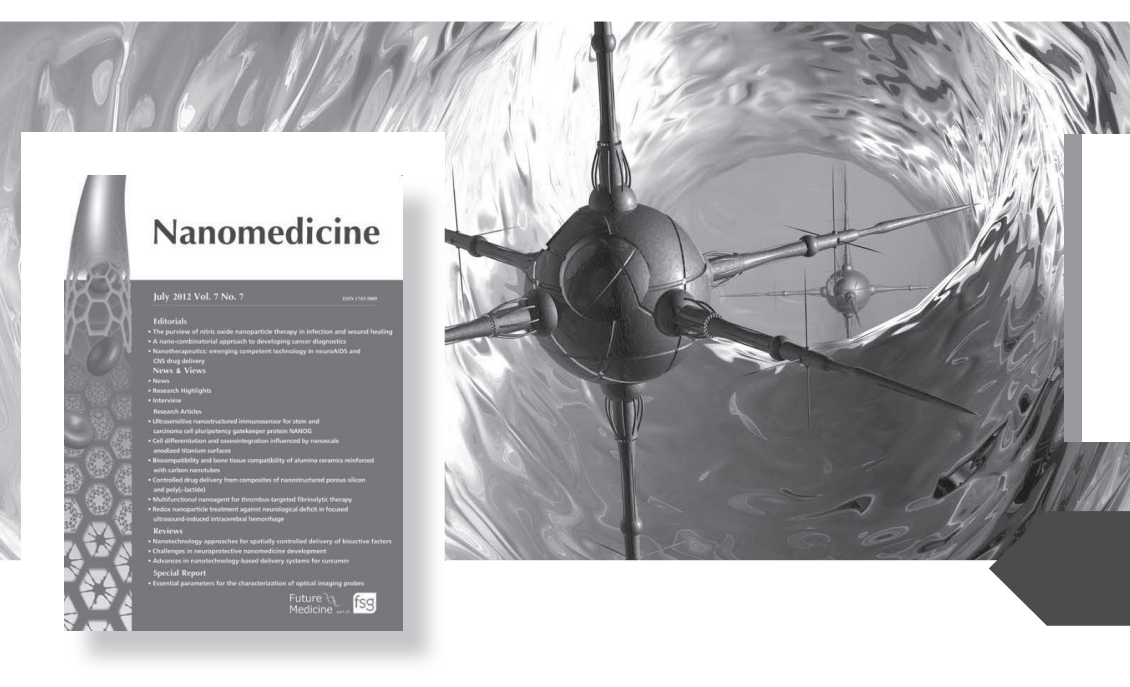

IMPACT FACTOR: 4.727 (2016)

\section{Nanomedicine}

ISSN: 1743-5889

Frequency per year: 24

www. futuremedicine.com 